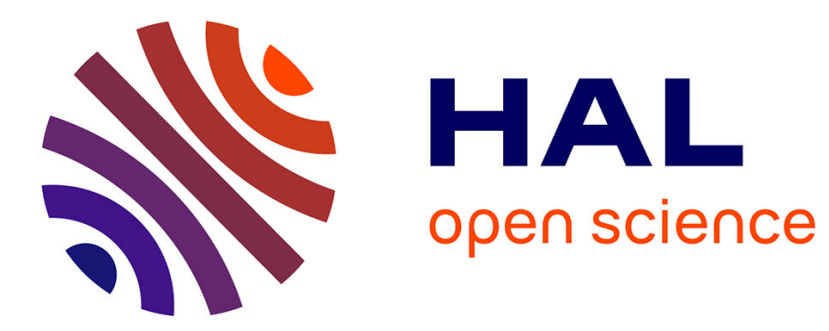

\title{
Microwave experiments using open chaotic cavities in the realm of the effective Hamiltonian formalism
}

Ulrich Kuhl, Olivier Legrand, Fabrice Mortessagne

\section{To cite this version:}

Ulrich Kuhl, Olivier Legrand, Fabrice Mortessagne. Microwave experiments using open chaotic cavities in the realm of the effective Hamiltonian formalism. Fortschritte der Physik / Progress of Physics, 2013, 61 (2 - 3), pp.404 - 419. 10.1002/prop.201200101 . hal-00745609

\section{HAL Id: hal-00745609 \\ https://hal.science/hal-00745609}

Submitted on 26 Oct 2012

HAL is a multi-disciplinary open access archive for the deposit and dissemination of scientific research documents, whether they are published or not. The documents may come from teaching and research institutions in France or abroad, or from public or private research centers.
L'archive ouverte pluridisciplinaire HAL, est destinée au dépôt et à la diffusion de documents scientifiques de niveau recherche, publiés ou non, émanant des établissements d'enseignement et de recherche français ou étrangers, des laboratoires publics ou privés. 


\title{
Microwave experiments using open chaotic cavities in the realm of the effective Hamiltonian formalism
}

\author{
Ulrich Kuhl, Olivier Legrand, and Fabrice Mortessagne \\ Université de Nice Sophia-Antipolis, Laboratoire de Physique de la Matière Condensée, CNRS UMR 7336, \\ 06108 Nice, France
}

Key words Scattering matrix, effective Hamiltonian, quantum chaos, classical wave experiments.

\begin{abstract}
The description of open quantum system successfully uses the concept of the effective Hamiltonian, which takes into account the coupling to the environment. The concept can be extended to classical waves. Experimentally, classical waves are very convenient as they allow to control boundaries, coupling and absorption. We combine here recent works with microwaves investigating properties of open systems which can be described by an effective Hamiltonian. This ranges from spectral properties like modifications to level dynamics, width distribution and coupling fidelity to spatial properties like intensity distributions and complexness parameter which describes the non-orthogonality of eigenfunctions.
\end{abstract}

Copyright line will be provided by the publisher

\section{Introduction}

Any measurement opens a wave system. This coupling to the continuum drastically changes the system properties by converting discrete energy levels into decaying resonance states and induces currents that did not exist in the closed system. One possible description is the effective Hamiltonian (see [1] for an overview). Thus typical quantities investigated in the realm of quantum chaos [2] have to be modified like nearest neighbor spacing distributions, avoided crossings, and new features occur like resonance width, resonance trapping and current flows. In particular, we will be interested in statistical properties in the case of chaotic systems.

In this manuscript we review experimental investigations of those properties using two dimensional microwave cavities. These experiments have the advantage that the time independent two dimensional Schrödinger equation and the corresponding Helmholtz equation are mathematically equivalent [2]. The experimental setup is simple and the main measurement device, a vector network analyser, is commercially available. The coupling to the environment, typically to the analyser, can be either performed by microwave waveguides, where each supported mode is an individual channel, or by coaxial antennas which typically only support a single mode. The antennas have the advantage that they perturb the closed system only weakly and they can be positioned arbitrarily in the two dimensional 'billiard'. Additionally they can operate over the whole frequency range, whereas waveguides have a lower cutoff frequency.

Microwave experiments have also induced the investigation of the effects of absorption on different quantities like the Poisson kernel and the width distribution. Absorption can be introduced into the effective Hamiltonian by attaching 'infinitely' many but weakly coupled channels additionally to the real channels. These absorptive channels have often similar effect as the dephasing probe introduced by Büttiker to describe the electron transport [3], like broadening the resonances, but differences can be found if their absorption or dephasing strength can be changed parametrically [4]. Additionally, there exists two type of absorptions in microwave cavities, one which can be described just by an additional constant or more precisely speaking weakly frequency depending width induced by top and bottom plates, and a mode dependent additional term coming from the boundary [5,6]. Therefore the measured scattering matrix is only subunitary. 
The measured quantities are reflection and transmission amplitudes through the introduced channels. Thus, all elements of the scattering matrix are experimentally directly accessible including the phases [2]. Also, the systems can be controlled easily down to the sub-wavelength regime. It is the purpose of this review to give an overview of different quantities using only a single description given in section 2 . In general the definition of the effective Hamiltonian and the scattering matrix elements differ by factors of $\pi$ or 2 in coupling constants and the normalization to the mean level spacing enters at different places. From the experimental point of view, the distribution of scattering matrix elements are better accessible than the resonances of the effective Hamiltonian. Often the resonances can only be extracted in the regime of isolated resonances, i.e. assuming only weak coupling. There are only a few results for strongly overlapping resonances, e.g. for the resonance trapping (see Subsec. 3.3 and [7, 8]) or for width distribution (see Subsec. 3.2 and [9]). Hopefully it will be possible in the future to extract the resonances reliably even in the strongly overlapping regime and follow them parametrically in the complex plane. Thus one could directly relate for example the already available results presented for the scattering fidelity (see Subsec. 3.4 and [10, 11]) or the Poisson kernel (see Subsec. 3.2) to the dynamics of the complex resonances experimentally.

In Sec. 2 we give a short introduction to the effective Hamiltonian. As the effective Hamiltonian cannot be measured directly we introduce the scattering matrix and relate it to the effective Hamiltonian. We fix our notation which will be used throughout the manuscript and might differ from the original papers. Experiments related to spectral properties are described shortly in the corresponding subsections of Sec. 3 . We start with the investigation of avoided crossings occurring for level dynamics under parametric changes of the system (Sec. 3.1). The Poisson kernel and the corresponding width distribution are presented in Sec. 3.2. The resonance trapping effect is investigated in Sec. 3.3 by increasing the coupling to the system using a microwave cavity with attached waveguide, and the fidelity created by a variation of coupling is shown in Sec. 3.4. Finally, we also present results on wave functions and measures of openness in Sec. 4 and then summarize the results and give an outlook in Sec. 5.

\section{Effective Hamiltonian and scattering theory}

\subsection{Effective Hamiltonian for chaotic system}

The general case of $M$ scattering channels connected to $N$ levels of the closed cavity can be described in terms of the following effective non-Hermitian Hamiltonian

$$
H_{\text {eff }}=H-i \sum_{c=1}^{M} \kappa_{c} W_{c} W_{c}^{\dagger} .
$$

Here, the internal Hamiltonian $H$ of the closed system is represented by a Hermitian $N \times N$ matrix, whereas $W_{c}$ are $M$ vectors of length $N$ containing the information on the coupling of the levels to the continuum through $M$ coupling channels. The $W_{c}$ are assumed to be normalized to one, $W_{c}^{\dagger} W_{c}=1$, and $\kappa_{c}$ is the coupling constant of channel $c$. Have in mind that there exists a scale induced by the Hamiltonian which is the mean level spacing $\Delta$.

Such an approach was initially developed in nuclear physics [12-14] and since then has been successfully applied to study various aspects of open systems, including wave billiards [2,15-17]. Usually, the phenomenological coupling constants $\kappa_{c}$ are considered as real numbers which enter the final expressions via the so-called transmission coefficients $T_{c}$. However, in the general case of an experiment it might be difficult to vary only the coupling of a channel without perturbing the system otherwise, thus leading to complex coupling coefficients $\kappa_{c}$. The only constraint is $\operatorname{Re}\left(\kappa_{c}\right) \geq 0$, due to the causality condition on the $S$-matrix. We note that quite a similar problem of nonzero $\operatorname{Im}\left(\kappa_{c}\right)$ arises in shell-model calculations due to the principal value term of the self-energy operator, cf. [12] and [13].

In this paper we will restrict ourself to open systems, where the classical counterpart of the wave system is fully chaotic and time reversal symmetry is respected. In this case, the Hamiltonian of the closed system is described by a large $N \times N$ random Gaussian orthogonal matrix (GOE), in which case, universal fluctuations are expected in the limit $N \gg 1$ over a scale of the order of the mean spacing $\Delta$. More specifically, 
by restricting the study to the center of the spectrum, the level spacing is given by $\Delta=\Lambda \pi / N$ where $2 \Lambda$ is the radius of the semicircle density for GOE [18]. Depending on the quantities of interest, the induced energy dependence on the imaginary part can be incorporated into the statistics of the closed Hamiltonian, i.e. $H^{\prime}=H-\Delta H_{i m}$ with $\Delta H_{i m}=\sum_{c=1}^{M} \operatorname{Im}\left(\kappa_{c}\right) W_{c} W_{c}^{\dagger}$. One should have in mind that the GOE assumption for $H^{\prime}$ is an approximation as the induced change of energy $\Delta H_{i m}$ is not described by GOE. In this paper $\Delta H_{i m}$ is only playing an important role in section 3.4, where we investigate the fidelity of the system under a change of the coupling.

The non-Hermiticity of $H_{\text {eff }}$ yields a set of complex eigenvalues $\left\{\mathcal{E}_{n}\right\}$ associated to two distinct sets of eigenvectors called left $\left\{\left\langle\psi_{n}^{L}\right|\right\}$ and right $\left\{\left|\psi_{n}^{R}\right\rangle\right\}$ eigenvectors:

$$
H_{\text {eff }}\left|\psi_{n}^{R}\right\rangle=\mathcal{E}_{n}\left|\psi_{n}^{R}\right\rangle, \quad\left\langle\psi_{n}^{L}\right| H_{\text {eff }}=\left\langle\psi_{n}^{L}\right| \mathcal{E}_{n},
$$

where the complex eigenvalue $\mathcal{E}_{n}=E_{n}-(i / 2) \Gamma_{n}$ gives respectively the energy $E_{n}$ and the resonance width $\Gamma_{n}>0$ of the $n$th resonance. The left and right eigenvectors, which describe the resonance states, satisfy the condition of bi-orthogonality, $\left\langle\psi_{n}^{L} \mid \psi_{m}^{R}\right\rangle=\delta_{n m}$ and completeness, $\sum_{n=1}^{N}\left|\psi_{n}^{R}\right\rangle\left\langle\psi_{n}^{L}\right|=\mathbb{1}$. In the context of microwave cavities, the validity of model $(1)$ has been established in previous works $[17,19]$. More general models of non-Hermitian Hamiltonian operators may be used to study open quantum systems (see e.g. the topical review written by I. Rotter [1]). This formalism has been successfully applied to wave billiards for which antennas and absorption are respectively described by physical and fictitious coupling channels [19-21].

\subsection{Scattering theory including the effective Hamiltonian}

According to the general scattering formalism [12,13,22], the resonance part of the $S$-matrix at the scattering energy $E$ can be expressed in terms of $H_{\text {eff }}$ as follows [11]:

$$
S_{a b}(E)=\delta_{a b}-2 i \sqrt{\operatorname{Re}\left(\kappa_{a}\right) \operatorname{Re}\left(\kappa_{b}\right)} W_{a}^{\dagger} \frac{1}{E-H_{\mathrm{eff}}} W_{b} .
$$

In the case where the coupling amplitudes change slowly with the energy, one may safely consider the complex eigenvalues $\mathcal{E}_{n}$ of $H_{\text {eff }}$ as the only singularities of the $S$-matrix in the complex energy plane.

Using random matrix theory (RMT) for large matrices at $E=0$, the transmission coefficients are given in terms of the average $S$-matrix diagonal elements $\left\langle S_{c c}\right\rangle=\left(1-\kappa_{c}^{*}\right) /\left(1+\kappa_{c}\right)$ by $[11,13,18]$

$$
T_{c} \equiv 1-\left|\left\langle S_{c c}\right\rangle\right|^{2}=1-\left|\frac{1-\kappa_{c}^{*}}{1+\kappa_{c}}\right|^{2}=\frac{4 \operatorname{Re}\left(\kappa_{c}\right)}{\left|1+\kappa_{c}\right|^{2}}, \quad c=1, \ldots, M .
$$

Thus $T_{c}$ is accessible directly from a reflection measurement and $\kappa_{c}$ can be determined uniquely if $\operatorname{Im}\left(\kappa_{c}\right)=$ 0 . It is worth noting that, in the case of real $\kappa_{c}$, one gets $T_{c}=\frac{4 \kappa_{c}}{\left(1+\kappa_{c}\right)^{2}} \in[0,1]$, so that $T_{c} \ll 1$ and $T_{c}=1$ correspond to an almost closed or perfectly open channel $c$, respectively. In the case of purely imaginary $\kappa_{c}$ corresponding to perfect reflection, the channel is closed, $T_{c}=0$. We will use this in the investigation of the coupling fidelity (see Subsec. 3.4 and [11]), where the effect of adding a channel (i.e. $T_{c}=0 \rightarrow T_{c}=1$ ) is investigated in the scattering fidelity.

Typically, the elements of the scattering matrix can be written as a sum of Lorentzians assuming that the coupling constants $\kappa_{c}$ have only a weak energy dependance within the resonance width, i.e. one is far from energy thresholds

$$
S_{a b}(E)=\delta_{a b}-i \sum_{n} \frac{a_{n}}{E-E_{n}+\frac{i}{2} \Gamma_{n}} .
$$

where $a_{n}$ is the complex amplitude, $E_{n}$ the eigenenergy and $\Gamma_{n}$ the width of the resonance. $\Gamma_{n}$ describes in the time domain the exponential decay of the $n$th eigenstate. In the weak coupling regime $(\kappa \ll 1$, assuming real $\kappa$ ), the resonances do not overlap and the non-Hermitian part of $H_{\text {eff }}$ can be treated as a 
perturbation of the Hermitian part $H$ and one can relate the parameters of the Lorentzians to the closed system. In this Breit-Wigner approximation the elements of the scattering matrix $S$ can be written as

$$
S_{a b}(E)=\delta_{a b}-2 i \sum_{n} \frac{\sqrt{\operatorname{Re}\left(\kappa_{a}\right) \operatorname{Re}\left(\kappa_{b}\right)} W_{a, n}^{\dagger} W_{b, n}}{E-E_{n}+\frac{i}{2} \Gamma_{n}} .
$$

Assuming real $\kappa$, to leading order in $\kappa$, the real parts of $\mathcal{E}_{n}$ are given by the eigenenergies of the closed system, $H|n\rangle=E_{n}|n\rangle$, while their widths are given by :

$$
\Gamma_{n}=2 \sum_{c=1}^{M} \kappa_{c}\left\langle n\left|W_{c} W_{c}^{\dagger}\right| n\right\rangle
$$

An investigation of the joint distribution of the complex reflection amplitude $S_{11}$ and the width $\Gamma_{n}$ is presented in Subsec. 3.2. Likewise, the right eigenvectors of $H_{\mathrm{eff}}$ read :

$$
\left|\psi_{n}^{R}\right\rangle=|n\rangle-i \sum_{c=1}^{M} \kappa_{c} \sum_{m \neq n} \frac{\left(W_{c} W_{c}^{\dagger}\right)_{m n}}{E_{n}-E_{m}}|m\rangle .
$$

We investigate the eigenvectors, i.e. the eigenfunctions of the system in section 4. Often one can use a $2 \times 2$ random matrix toy model to obtain analytic results. An example is shown in Subsec. 3.1.

\section{Classical wave experiments on spectral properties}

\subsection{Level dynamics}

It is often relevant to study the dynamics of levels when a parameter of the system is varied. For a completely uncorrelated spectrum typical of a system with regular dynamics, levels evolve ignoring the presence of others. It ensues that for certain values of the parameter, levels may become degenerate. On the contrary, in a chaotic system, level repulsion precludes such a possibility. To characterize the parametric dynamics of levels one may consider the statistics of local minima of level spacings, also called avoided crossings. In this section, following [23], we recall how a two-level model with a large number of open decay channels can be used to describe avoided level crossing statistics in open chaotic billiards. This model allows us to describe the fundamental changes of the probability distribution of the avoided level crossings compared with the case of a closed wave system [24]. In particular, it is found that the decay process due to open channels induces a modification of the probability distribution of the avoided level crossings at small spacings due to an attraction of the resonances. In a microwave experiment presented in [25] the spectral properties of a superconducting two-dimensional microwave billiard were investigated, where the geometry of the cavity is varied by rotating a dielectric (Teflon) wedge creating an evolution of the levels (see inset of Fig. 1). The resonator modeled an open quantum billiard whose classical dynamics is chaotic with the antennas acting as single scattering channels [23]. The influence of the flux of microwave power was so weak that it could not be detected through spectral quantities like the nearest neighbor spacing distribution or the spectral rigidity at a fixed value of the parameter. But the distribution of the avoided crossings is more sensitive to small effects showing clear deviations from the GOE result, which could be attributed to the openness of the cavity.

In reference [23], an effective Hamiltonian $H_{\text {eff }}(\mu)$ (see Eq. (1)) which depends on a continuous parameter $\mu$ through its Hermitian part $H(\mu)$ is used [25]. The Hamiltonian $H(\mu)$ of the closed system is modeled by a $2 \times 2$ random matrix and the coupling to the environment in terms of $M$ equivalently coupled open channels $\left(\kappa_{c} \equiv \kappa\right)$. The eigenvalues of the effective Hamiltonian are complex and read $\epsilon_{ \pm}=E_{ \pm}-(i / 2) \Gamma_{ \pm}$, where $E_{ \pm}$and $\Gamma_{ \pm}$are, respectively, the two eigenenergies and the two spectral widths of the 2-level model. For the study of statistical properties $H(\mu)$ is replaced by a Gaussian random matrix [2] and the coupling matrix elements $\sqrt{\kappa} W_{c, n}$ are chosen to be real Gaussian-distributed with zero 
mean and variance $\sigma^{2}=\kappa \Delta / \pi$, where $\Delta$ is the mean level spacing of the closed system [5]. In the eigenbasis of $H(\mu)$ (the $\mu$-dependence will be omitted in the following), the effective Hamiltonian is written as

$$
H_{\text {eff }}=\left(\begin{array}{cc}
E_{1} & 0 \\
0 & E_{2}
\end{array}\right)-\frac{i}{2}\left(\begin{array}{cc}
\Gamma_{11} & \Gamma_{12} \\
\Gamma_{21} & \Gamma_{22}
\end{array}\right)
$$

where $E_{1,2}$ are the $\mu$-dependent eigenenergies of $H\left(E_{2}>E_{1}\right.$ is assumed) and $\Gamma_{n p}=\kappa \sum_{c=1}^{M} W_{c, n} W_{c, p}$.

The complex eigenvalues of the effective Hamiltonian $H_{\text {eff }}$ in Eq. (9) read

$$
\epsilon_{ \pm}=\frac{E_{1}+E_{2}-\frac{i}{2}\left(\Gamma_{11}+\Gamma_{22}\right) \pm \sqrt{D}}{2} \text { with } D=\left(\left(E_{1}-E_{2}\right)+\frac{i}{2}\left(\Gamma_{22}-\Gamma_{11}\right)\right)^{2}-\Gamma_{12} \Gamma_{21} .
$$

Thus the spacing between the two eigenenergies is given by $d=\epsilon_{+}-\epsilon_{-}=\operatorname{Re}(\sqrt{D})$. Considering the limit of a large number of open channels $M$ in the weak coupling regime, we may apply the central limit theorem and replace the random variables depending on the coupling amplitudes by their averages $\left\langle\Gamma_{n n}\right\rangle=M \sigma^{2}$ and $\left\langle\Gamma_{n p} \Gamma_{p n}\right\rangle=M \sigma^{4}$. Then the spacing $d$ is given by

$$
d=\left\{\begin{array}{ll}
\sqrt{s^{2}-M \sigma^{4}} & \text { if } s>\sqrt{M} \sigma^{2} \\
0 & \text { otherwise }
\end{array},\right.
$$

where $s=E_{2}-E_{1}$ is the spacing of the eigenenergies of the closed system. Note that since $M \sigma^{4}=\operatorname{var}(\Gamma)$, it means that the changes of the spacings due to the openness of the system are related to the fluctuations of the spectral widths [26]. In the limit $M \rightarrow \infty$ and $\sigma^{2} \rightarrow 0$ with $M \sigma^{2}=\langle\Gamma\rangle$ fixed, $\operatorname{var}(\Gamma) \rightarrow 0$ and thus the spacing between eigenenergies of the open system converges to that of the closed system, $d \rightarrow s$, in spite of non-vanishing losses. This implies that the values of the parameter $\mu$ at the avoided crossings are the same for the closed and the open system. Thus, to obtain the distribution of avoided crossings $c$ of the open system, the spacings $s$ are assumed to be distributed as the avoided crossings of the corresponding closed system. With Eq. (11) the probability distribution of the avoided level crossings $p(c)$ is given by

$$
p(c)=\left\langle\delta(c) \theta\left(\sqrt{M} \sigma^{2}-s\right)\right\rangle+\left\langle\delta\left(c-\sqrt{s^{2}-M \sigma^{4}}\right) \theta\left(s-\sqrt{M} \sigma^{2}\right)\right\rangle,
$$

where $\theta$ is the Heaviside step function and the triangular brackets denote averaging with respect to the spacing $s$.

Using the probability distribution of avoided crossings for (closed) chaotic systems with time reversal symmetry calculated by Zakrzewski and Kuś [24], $p(s)=\sqrt{\frac{2}{\pi \alpha^{2}}} e^{-s^{2} /\left(2 \alpha^{2}\right)}$, where the mean value of $s$ is given by $\langle s\rangle=\alpha \sqrt{2 / \pi}$, and averaging over $s$ yields

$$
p(c)=\operatorname{erf}\left(\frac{\sqrt{M} \sigma^{2}}{\sqrt{\pi} \alpha}\right) \delta(c)+\sqrt{\frac{2}{\pi \alpha^{2}}} \frac{c e^{-\left(c^{2}+M \sigma^{4}\right) /\left(2 \alpha^{2}\right)}}{\sqrt{c^{2}+M \sigma^{4}}},
$$

where $\alpha$ fixes the average of $c$. Note that the linear behavior of the distribution $p(c)$ at small spacings induces a dip and the local minima of the spacings have a zero-crossings contribution leading to the presence of a $\delta$-peak at the origin.

In the experiment described in [25], only finite spacings could be measured due to the finite frequency resolution implied by the discrete sampling of the data. Therefore, it is more convenient to consider the distribution of nonvanishing avoided crossings (the second term in the right-hand-side of (13) with proper normalization) to compare theory and experiment. The experimental results of [25] were obtained using a superconducting microwave cavity, thus minimizing dissipative processes. Three antennas were attached to the cavity: they correspond, in our model, to $M=3$ open channels [27]. Absorption into the walls could be safely neglected in the analysis of the experimental data. The analytic result obtained through a 


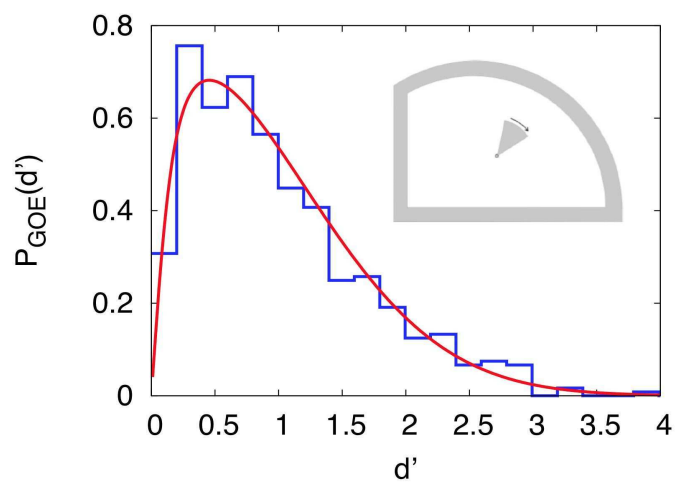

Fig. 1 Probability distribution of non vanishing avoided crossings. In blue, the experimental histogram ( [25]). In red the analytical prediction with $\kappa_{f i t}=0.182$ (obtained through a least square procedure with $M=3$ ). Inset: schematic representation of the cavity with a rotating Teflon wedge (see [25]).

fit using $\kappa$ as a parameter (continuous curve) is shown in Fig. 1. The 2-level model result follows closely the experimental histogram. This provides a clear confirmation of the interpretation drawn in [25] that the deviation of the avoided-crossings distribution from the predicted GOE result for closed systems was due to the measurement process, i.e. the influence of the three antennas, which couple the cavity modes inside the resonator to the outside.

In other microwave experiments, exceptional points (EP) have been investigated [28,29]. At an EP, two or more eigenvalues and also the associated eigenvectors coalesce $[1,30]$. To investigate the EP, resonances are moved in the complex plane by varying two parameters. Typically one parameter is mainly inducing a shift of the real part, whereas the other corresponds to an imaginary perturbation inducing a change of resonance width. Let us assume now a typical EP which involves only two eigenvalues. By encircling the EP parametrically once the two resonances are exchanged while only one wave function acquires a sign. Encircling a second time another exchange occurs but the two wavefunctions differ now by a phase of $\pi$ from the starting situation. Finally the original situation is restored when encircling the EP 4 times [31]. This behavior has been demonstrated using microwave cavities [28, 29].

\subsection{Poisson kernel and width distribution}

Often, one cannot extract the resonances and a direct investigation of the effective Hamiltonian is not accessible, but information of the $S$-Matrix like reflection and transmission amplitudes are. As we are interested not in the individual properties of the system but in statistical properties, the distribution of amplitudes and phases are of interest. In this section we will restrict to a system coupled only via a single channel to the environment and rely on the references [20,21]. The statistical distributions of the $S$-matrix elements with imperfect coupling have been addressed by several authors [32-38]. Experimentally not only the direct processes have to be taken into account but also losses or absorption. When absorption is present, $S$ is a subunitary matrix and in the single-channel case the $S$ matrix can be parametrized using the reflection coefficient $R$ as $S=\sqrt{R} e^{i \theta}$ The coupling between the scattering channels and the interior region is given by the transmission coefficient $T_{a}=1-|\langle S\rangle|^{2}$ (see Eq. 4). The subindex $a$ will be used to denote the antenna coupling. The distribution $P_{R, 0}(R)$ of $R$ is known [17,39] and there exists an analytical approximation for $\operatorname{GOE}(\beta=1)$ which we will use here [21]

$$
P_{R, 0}\left(R_{0}\right)=C \frac{e^{-\alpha /(1-R)}}{(1-R)^{2+1 / 2}}\left[A \alpha^{-1 / 2}+B(1-R)^{1 / 2}\right],
$$

where $\alpha=\gamma / 2, A=\alpha\left(e^{\alpha}-1\right), B=\left(1+\alpha-e^{\alpha}\right), C=\alpha^{3 / 2} / \Gamma(3 / 2)$ and $\Gamma$ is the upper incomplete Gamma function. $\gamma$ is the absorption strength, e.g. the exponentiel decay rate of the intensity, inside the 
(a)
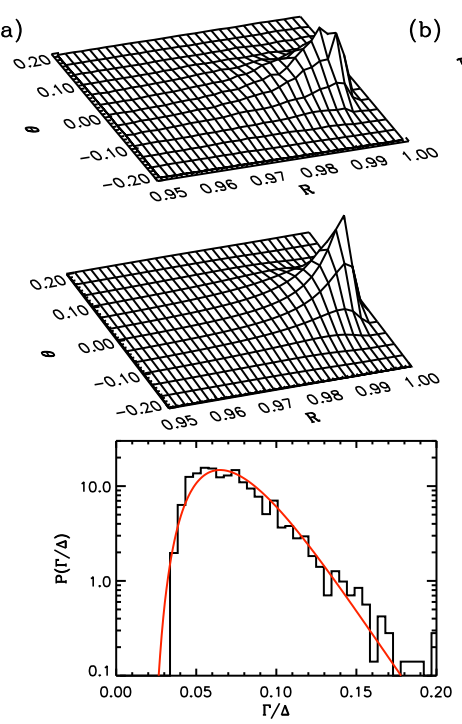

(b)
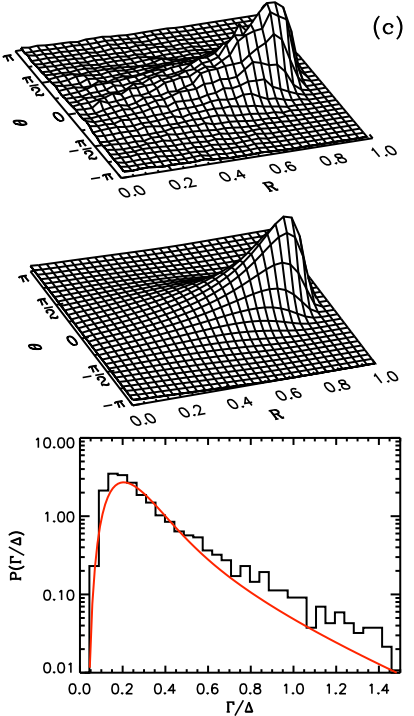

(c)
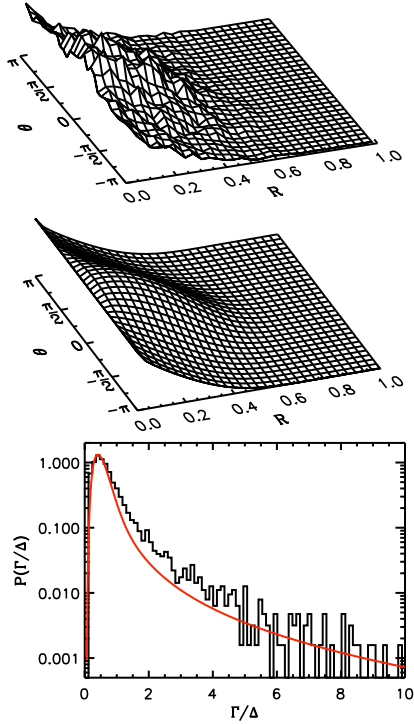

Fig. 2 Joint distribution $P(R, \theta)$ of the $S$-matrix for different coupling and absorption regimes: (a) $\gamma=0.56$, $T_{a}=0.116$ (weak absorption-weak coupling) (b) $\gamma=2.42, T_{a}=0.754$, and (c) $\gamma=8.40, T_{a}=0.989$ (stronger absorption-nearly perfect coupling). The upper, middle rows correspond to experiment and theory, respectively (see [21]). Note the change of scales for $R$ and $\theta$. Below the corresponding normalized width distribution is shown. The black histogram was obtained from the experiment using the harmonic inversion. The solid line corresponds to the theoretical prediction from [17] taking additionally fluctuating absorption into account [41] (see [21,41]).

closed system. We denote a system with absorption strength $\gamma$ and perfect coupling $\left(T_{a}=1\right)$ by $S_{0}=$ $\sqrt{R_{0}} e^{i \theta_{0}}$. Introducing the $S$-matrix of the antenna [36,40] and using the combination rule of $S$-matrices, the distribution $P_{S}(S)$ for the system including direct processes was derived as [21]

$$
P_{S}(S)=\left|\frac{\partial\left(R_{0}, \theta_{0}\right)}{\partial(R, \theta)}\right| P_{S, 0}\left(S_{0}\right)=\left(\frac{1-\langle S\rangle^{2}}{|1-S\langle S\rangle|^{2}}\right)^{2} \frac{1}{2 \pi} P_{R, 0}\left(R_{0}\right)
$$

The experimental realization using microwaves has been performed by a rectangular cavity where a movable half circle is attached to one wall. The microwaves excite the cavity via a single antenna, where the antenna coupling is frequency dependent. Details of the experimental description can be found in Refs. [9, 20,21]. In Fig. 2 the joint probability $P(S)=P(R, \theta)$ is shown for three different absorption and coupling regimes (columns a-c). In the upper row the experimental distributions and in the middle row the theoretical distributions are shown. A good agreement is found especially if one takes into account that all parameters of the theory can be fixed in advance by average quantities.

To make a more direct relation to the effective Hamiltonian, we will now investigate the resonances, more precisely the distribution of resonance width $\Gamma$. From the previous reflection measurements the poles of the $S$-matrix in the complex plane have been determined [41]. The resonances have been extracted by means of the harmonic inversion [42]. Thus it became possible to resolve the resonances in a regime where the line widths exceed the mean level spacing up to a factor of 10. The obtained experimental distributions of line widths are shown in the lower row of Fig. 2. The distributions are in agreement with predictions from random matrix theory [17] when wall absorption and fluctuations caused by couplings to additional absorbing channels are considered [41].

The joint probability distributions $P(R, \theta)$ shown in the upper row of Fig. 2 is induced by the resonances and eigenfunctions of the effective Hamiltonian. The presented width distribution shown in the lower part 
is only one part necessary to generate the $P(R, \theta)$. The other parts are the distribution of the resonances including their correlations to the widths and the value of eigenfunctions at a fixed position $r$ (antenna position). It will be a future challenge to derive all this correlations theoretically and experimentally.

In a recent work the same data has been used to investigate the Wigner surmise of the nearest neighbor spacing for an open system [43]. Using the effective Hamiltonian approach in the $2 \times 2$ approximation (see Sec. 3.1) the authors were able to derive an analytical formula and an agreement to the experimental nearest neighbor spacing distribution was found [43].

In this subsection we have shown the width distribution for different coupling strength. In the following subsection we will investigate how the resonances, especially their widths, will change when gradually changing the coupling to a single channel.

\subsection{Resonance trapping}

Resonance trapping is a phenomenon appearing in strongly open quantum systems. We will present results from $[7,8]$ using a slightly different notations. For details refer to the original works. Resonance trapping is caused by the interaction of overlapping resonance states via the continuum of scattering states by which some of the states align with the channels by trapping other ones. Therefore the total coupling strength is given by

$$
\Gamma_{\text {tot }}=\sum_{n=1}^{N} \Gamma_{n} \approx \Gamma_{\text {align }}=\sum_{n=1}^{M} \Gamma_{n} \text { i.e. } \quad \Gamma_{\text {trap }}=\sum_{\mathrm{n}=\mathrm{M}+1}^{\mathrm{N}} \Gamma_{\mathrm{n}} \approx 0,
$$

where $N$ is the total number of states and $M$ states are strongly coupled to the environment. Due to the reordering processes, the other $N-M$ states are only weakly coupled to the environment. Resonance trapping can be observed if the total coupling strength is varied.

Starting from weak coupling where the resonance states are still isolated, we have Breit-Wigner resonances (see Eq. 6) and the Hamiltonian $H_{\text {eff }}$ is almost diagonal. Increasing the coupling the resonances start to overlap and a redistribution in the spectrum of the system takes place due to the non-diagonal terms of the non-Hermitian part $\kappa W W^{\dagger}$ of $H_{\mathrm{eff}}$. The Hamiltonian $H_{\mathrm{eff}}$ has to be diagonalized,

$$
H_{\mathrm{eff}}\left|\tilde{\psi}_{n}\right\rangle=\left(\tilde{E}_{n}-\frac{i}{2} \tilde{\Gamma}_{n}\right)\left|\tilde{\psi}_{n}\right\rangle .
$$

leading to

$$
S_{a b}(E)=\delta_{a b}-2 i \sum_{n} \frac{\sqrt{\operatorname{Re}\left(\tilde{\kappa}_{a}\right) \operatorname{Re}\left(\tilde{\kappa}_{b}\right)} \tilde{W}_{a, n}^{\dagger} \tilde{W}_{b, n}}{E-\tilde{E}_{n}+\frac{i}{2} \tilde{\Gamma}_{n}} .
$$

where $\sqrt{\operatorname{Re}\left(\tilde{\kappa}_{a}\right)} \tilde{W}=\left\langle\sqrt{\operatorname{Re}\left(\kappa_{a}\right)} W \mid \tilde{\psi}_{n}\right\rangle . \quad \tilde{W}, \tilde{E}_{n}$ and $\tilde{\Gamma}_{n}$ are generally energy dependent and $\tilde{W}$ is complex. The poles of the $S$ matrix are obtained from the solutions of the fixed-point equations

$$
E_{n}=\left.\tilde{E}_{n}\right|_{E=E_{n}} \quad \text { and } \quad \Gamma_{\mathrm{n}}=\left.\tilde{\Gamma}_{\mathrm{n}}\right|_{\mathrm{E}=\mathrm{E}_{\mathrm{n}}} .
$$

which determine the energies $E_{n}$ and widths $\Gamma_{n}$ of the resonance states. The eigenfunctions $\tilde{\psi}_{n}$ of $H_{\text {eff }}$ are bi-orthogonal. The resonances are no longer of Breit-Wigner type because of the energy dependencies of $\tilde{W}$ and $\tilde{\Gamma}_{n}$ (see $[44,45]$ ). The energy dependencies ensure the unitarity of the $S$ matrix.

This effect was demonstrated by a microwave experiment [7,8], where the coupling to the cavity via a waveguide was varied by a slit (see inset of Fig. 3). The reflection was measured via a waveguide of width $D=23.2 \mathrm{~mm}$ where a slit was positioned at distance $L=16 \mathrm{~mm}$ from the attachment to the cavity. The cavity has a form of a quarter Sinai shape with length $a=285 \mathrm{~mm}$, width $b=200 \mathrm{~mm}$, and radius of the quarter circle of $r=70 \mathrm{~mm}$. The slit opening $d$ was varied in steps of $0.1 \mathrm{~mm}$ and the resonance 


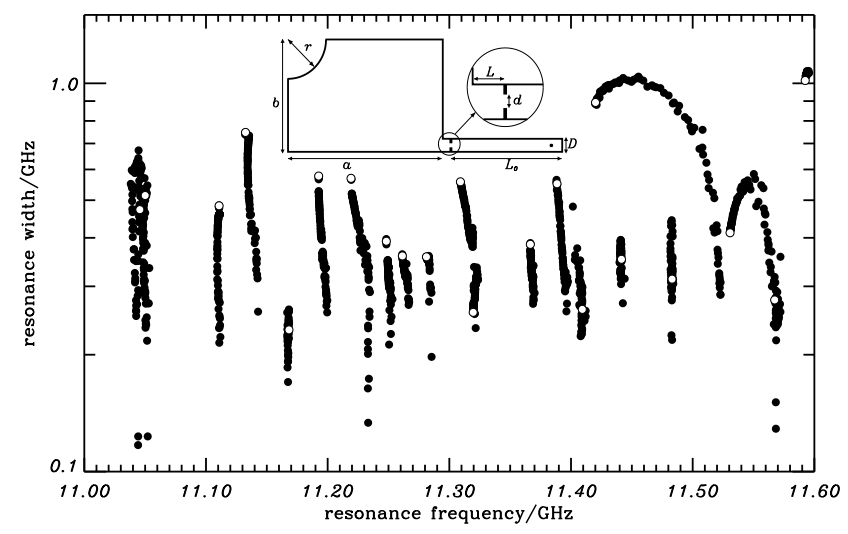

Fig. 3 Eigenvalues, obtained by fitting the experimental reflection data to Lorentzians, for slit position $L=16 \mathrm{~mm}$. The open circles denote the eigenvalues by full opening $(d=D)$. The inset shows a sketch of the billiard with attached waveguide and slit within the waveguide $\left(a=285 \mathrm{~mm}, b=200 \mathrm{~mm}, r=70 \mathrm{~mm}, L_{0}=204 \mathrm{~mm}, D=23.2 \mathrm{~mm}\right.$, $L=16 \mathrm{~mm})($ see $[8])$.

extracted by fitting a superposition of Lorentzians. In Fig. 3 the resonances in the frequency range from 11 to $11.6 \mathrm{GHz}$ are shown as a function of the slit opening. The open circles denote the eigenvalues by full opening $(d=D)$. Clearly a trapping effect is visible for several resonances, where the width $\Gamma$ for the full opening is smaller than the maximum width observed for smaller openings. For the cavity parameters roughly a quarter of all resonances investigated are trapped. The observed frequency shift in Fig. 3 is due to both the real and imaginary part of $\kappa$.

It is worthwhile to note that the width distribution once the resonance trapping has happened contains two contributions. One distribution $P\left(\Gamma_{\text {trap }}\right)$ of the trapped states which is similar to using a corresponding weak coupling and another part $P\left(\Gamma_{\text {align }}\right)$ of the broad aligned states. In the previous subsection the width distribution of the trapped states is shown, but in the case of the joint probability distribution $P(R, \Theta)$ both contributions are included (see Fig. 2c).

\subsection{Scattering Fidelity for varying coupling}

In this section we want to investigate the effect of opening the system by adding one additional channel, where another channel is used to measure the scattering matrix. We present here only a short theoretical description, which follows [11] (refer to this reference for details of the theory). In contrast to [11] we will compare here the perfectly reflecting to the perfectly absorbing channel, whereas in [11] a variation of the reflection was presented.

The quantity we want to use is the scattering fidelity [10] which is related to the fidelity [46, 47]. Both measure the stability of a wave system against perturbations. The fidelity is the time dependence of the overlap of the same initial pulse propagated by a 'slightly' different Hamiltonian, whereas the scattering fidelity amplitude $f_{a b}$ corresponds to the properly normalized spectral cross correlation function of scattering matrix elements $S_{a b}$ for 'slightly' different systems [10]:

$$
f_{a b}(t)=\frac{\left\langle\hat{S}_{a b}(t) \hat{S}_{a b}^{\prime *}(t)\right\rangle}{\sqrt{\left\langle\hat{S}_{a b}(t) \hat{S}_{a b}^{*}(t)\right\rangle\left\langle\hat{S}_{a b}^{\prime}(t) \hat{S}_{a b}^{\prime *}(t)\right\rangle}} .
$$

This definition ensures that $f_{a b}(0)=1$. Furthermore, an overall decay of the correlation functions due to absorption or other open channels drops out, provided the decay is the same for the parametric crosscorrelation functions in the nominator and the autocorrelation functions in the denominator. The scattering 
fidelity itself is

$$
F(t)=\left|f_{a b}(t)\right|^{2}
$$

For chaotic systems and weak coupling of the measuring antenna the scattering fidelity approaches the ordinary fidelity [48]. The time $t$ is given in units of the Heisenberg time $t_{H}=2 \pi \hbar / \Delta$, with $\Delta$ being the mean level spacing.

The scattering fidelity presented here will be denoted as coupling fidelity. We present here a sketch of an exact RMT prediction (for details see [11]). The coupling constant of the added channel will be denoted by $\kappa$ for $\hat{S}_{a b}(t)$ and $\kappa^{\prime}$ for $\hat{S}_{a b}^{\prime *}(t)$. The derivation is general but later the reference system is the system without channel, i.e. $\kappa^{\prime}=0$. For the case where $\kappa=\kappa^{\prime}$ the parametric correlation functions $\left\langle\hat{S}_{a b}(t) \hat{S}_{a b}^{\prime *}(t)\right\rangle$ in Eq. (20) reduces to the autocorrelation function, which is given by the famous Verbaarschot-WeidenmüllerZirnbauer (VWZ) integral [13]. For the case of $\kappa \neq \kappa^{\prime}$ the correlator $\left\langle\hat{S}_{a b}(t) \hat{S}_{a b}^{\prime *}(t)\right\rangle$ yields [11]

$$
\left\langle\hat{S}_{a b}(t) \hat{S}_{a b}^{\prime *}(t)\right\rangle=\left\langle\hat{S}_{a b}^{\mathrm{eff}}(t) \hat{S}_{a b}^{\mathrm{eff} *}(t)\right\rangle .
$$

Thus the parametric cross correlation function reduces to an autocorrelation function $\left\langle\hat{S}_{a b}^{\text {eff }}(t) \hat{S}_{a b}^{\text {eff } *}(t)\right\rangle$ given by the VWZ integral, where the transmission coefficient (Eq. (4)) of the varied channel has to be replaced by

$$
T^{\mathrm{eff}}=\frac{2\left(\kappa+\kappa^{\prime *}\right)}{(1+\kappa)\left(1+\kappa^{\prime *}\right)} .
$$

One can interpret $T^{\mathrm{eff}}$ as an effective transmission coefficient due to a parametric variation of the coupling strength in the varied channel. For $\kappa=\kappa^{\prime}$, the effective transmission coefficient $T^{\text {eff }}$ becomes equal to the conventional transmission coefficient (Eq. (4)). In contrast to the transmission coefficient in Eq. (4) the effective transmission coefficient $T^{\mathrm{eff}}$ is generally complex. The subsequent evaluation of the coupling fidelity cannot be done analytically and will be performed numerically.

As in our case only one channel with a coupling strength that depends on frequency is added, and the reflection is measured using the only other channel, i.e. via an attached antenna, the theoretical description of the scattering fidelity can be simplified. By denoting " $c$ " the added antenna and " $a$ " the measuring antenna, the effective Hamiltonian $H_{\text {eff }}$ may be written $H_{\text {eff }}=H_{\text {eff }}^{a}-i \kappa_{a} W_{a} W_{a}^{\dagger}$ where $H_{\text {eff }}^{a}$ is defined as [11]

$$
H_{\mathrm{eff}}^{a}=H-i \kappa_{c} W_{c} W_{c}^{\dagger}
$$

Note that the coupling constant $\kappa_{a}$ is generally complex and takes into account the effects of both the channel coupling and any reflection and phase shifts coming from the imperfect experimental environment, e.g. slight mismatches of cable analyzer connection etc.

Through this description, the $2 \times 2$ scattering matrix for both channels has thus been reduced to a $1 \times 1$ scattering matrix for the measuring antenna only

$$
S_{a a}=\frac{1-i \kappa_{a}^{*} W_{a}^{\dagger} \frac{1}{E-H_{\mathrm{eff}}^{a}} W_{a}}{1+i \kappa_{a} W_{a}^{\dagger} \frac{1}{E-H_{\mathrm{eff}}^{a}} W_{a}}
$$

As a basis system for the measurement we use a chaotic quarter Sinai cavity with length $l=472 \mathrm{~mm}$, width $w=200 \mathrm{~mm}$ and a quarter-circle of radius $r=70 \mathrm{~mm}$. Additional elements were inserted to reduce the influence of bouncing balls and a rotatable ellipse was introduced close to the center for ensemble averaging. The two antennas are situated such that there is no direct view of each other. The added perturbing antenna is directly attached to a vector network analyzer, mimicking an infinitely long channel. 

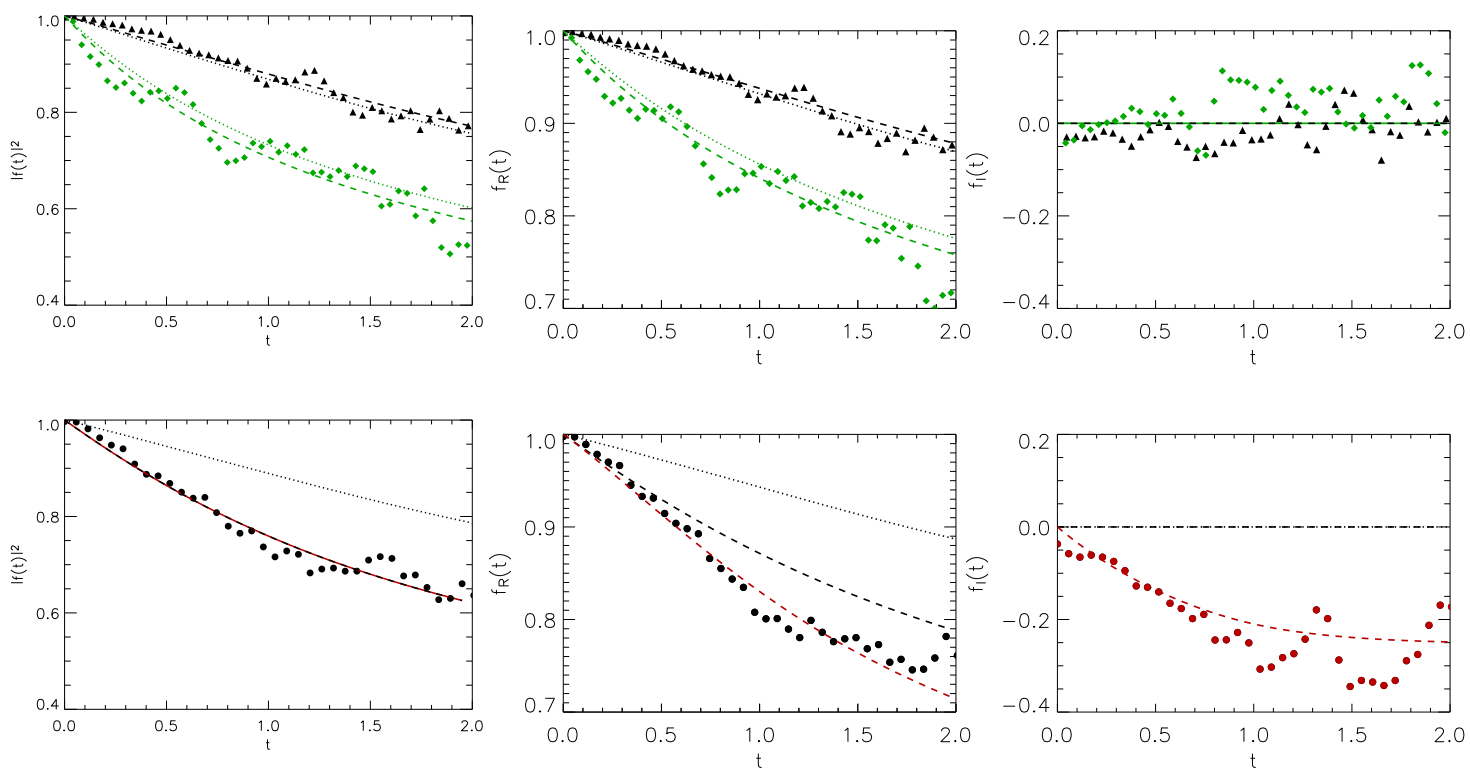

Fig. 4 Fidelity $|f(t)|^{2}$, real $f_{R}(t)$, and imaginary part $f_{I}(t)$ of the fidelity amplitude. Filled symbols show the experimental results and the theoretical curves are dotted for experimental parameter and dashed for fitting parameter. In the upper row the chosen frequency ranges are: $8.7-9.2 \mathrm{GHz}$ (black triangles) with $\kappa_{c}^{\text {exp }}=0.24, \kappa_{c}^{\text {fit }}=0.21$ and $9.8-10.3 \mathrm{GHz}$ (green diamonds) with $\kappa_{c}^{\exp }=0.43, \kappa_{c}^{\mathrm{fit}}=0.46$. In the lower row the frequency range is $7.2-7.7 \mathrm{GHz}$. Filled circles show the experimental results. The theoretical curves are dotted for experimental parameter $\kappa_{c}^{\text {exp }}=0.19$; dashed black for real valued fitting parameter $\kappa_{c}^{\text {fit }}=0.37$ and dashed red for complex valued fitting parameter $\kappa_{c}^{\mathrm{fit}, \mathrm{im}}=0.19+i 0.20$.

This gives us also the possibility to measure directly the reflection at the added channel $S_{c c}$ and calculate the coupling strength $T_{c}=1-\left|\left\langle S_{c c}\right\rangle\right|^{2}$, where the average is taken over different ensembles and a small energy window. Assuming that the coupling $\kappa_{c}$ has only a real part we can determine $\kappa_{c}$ uniquely from $T_{c}$.

In the upper part of Fig. 4 the fidelity introduced in Eq. (21) is plotted for two frequency ranges corresponding to two different coupling strengths $\kappa_{c}$. Using the experimentally determined $\kappa_{c}$ here denoted as coupling parameter $\kappa_{c}^{\text {exp }}$, a good agreement between experimental results (filled symbols) and theoretical curves (dotted line) is found without any fit. A fit of $\kappa_{c}$ to the experimental curves to the scattering fidelity $|f(t)|^{2}$ (the corresponding values are denoted by $\kappa_{c}^{\mathrm{fit}}$ ) which is plotted as dashed line shows only a minor improvement for the correspondence between experiment and theory. In both cases an agreement is found.

In the lower part of Fig. 4 we present the fidelity decay for the frequency range $7.2-7.7 \mathrm{GHz}$. Here we find an obvious deviation between the experimental results (filled circles) and the theoretical curve based on the experimental parameter $\kappa_{c}^{\exp }$ (dotted line). To determine the decay of the experimental fidelity decay we tried two possible ways of fitting $\kappa_{c}$ to the experimental data. The first is fitting $|f(t)|^{2}$ using a real valued $\kappa_{c}^{\mathrm{fit}}$ and the second is fitting the complex $f(t)$ using a complex $\kappa_{c}^{\mathrm{fit}}$,im.

While for the fidelity decays both fitting procedures lead to a good agreement between experiment and theory, the corresponding real $f_{R}(t)$ and imaginary $f_{I}(t)$ parts of the fidelity amplitude presented in Fig. 4 is described satisfyingly only by the red dashed curve, which is generated with the complex valued fitting parameter $\kappa_{c}^{\mathrm{fit}, \mathrm{im}}$. Thus in the frequency range from $7.2-7.7 \mathrm{GHz}$ the attached antenna cannot be described solely by an additional coupling but it has a non negligible scattering part inside the billiard. It is worthwhile to note that the real part of $\kappa_{c}^{\mathrm{fit}}$,im is matching the experimental $\kappa_{c}^{\exp }$ thus showing a consistent description. In case of the two other ranges shown in Fig. 4 the imaginary part is small and $\operatorname{Im}\left(\kappa_{c}\right)=0$ is a reasonable assumption. 


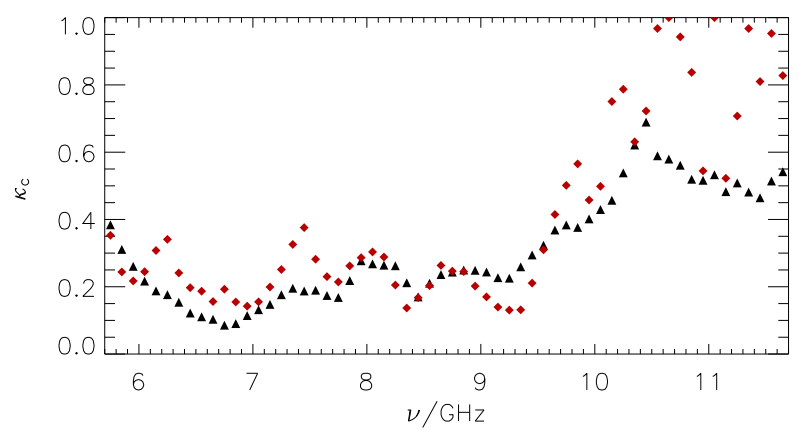

Fig. 5 Coupling constant $\kappa_{c}$ as a function of the mean frequency $\nu$; determined from the experimental transmission coefficient $T_{c}$ (black triangles) and as real valued fitting parameter to the experimental fidelity decay (red diamonds).

In Fig. 5 we plotted the coupling constant $\kappa_{c}$ determined from the experimental transmission coefficient $T_{c}$ as $\kappa_{c}^{\exp }$ (black triangles) and from the fit to the experimental fidelity decay $\kappa_{c}^{\text {fit }}$ (red diamonds). For frequencies from about $6 \mathrm{GHz}$ up to $10 \mathrm{GHz}$ the experimentally determined coupling constant and the fitting parameter do not deviate much from each other apart from some significant peaks like the one around $7.5 \mathrm{GHz}$ which results from system specific features, probably antenna resonances. Beyond $11 \mathrm{GHz}$ the way of determining the coupling constant via a reflection measurement at the perturbing antenna $c$ does not lead to convincing results. This might be due to the resonance trapping effect which was observed in similar microwave billiards (see section 3.3 and [7,8]). If the coupling of the newly attached channel is sufficiently strong, thus leading to a reorientation of the eigenvalues, an imaginary part of the coupling constant has to be taken into account. Typically the coupling constant and antenna transmission $T_{c}$ increase with frequency and above $10 \mathrm{GHz} T_{c}>0.8$. Thus in the regime of resonance trapping, taking the complex coupling coefficient into account is crucial for the coupling fidelity.

\section{Classical wave experiments on spatial properties}

\subsection{Wave functions}

We now turn to examine the consequences of openness on the spatial properties of the field.

While field statistics of open chaotic systems have been systematically studied for a given energy and considering the energy as a continuous parameter (see [21] and references therein), statistics of resonance states i.e. left and right eigenvectors of $H_{\text {eff }}$ are less understood. The impact of the openness is to turn real eigenfunctions into complex internal wavefunctions associated to resonances. This complexness being uniquely related to the presence of currents inside the system $[49,50]$. In order to quantify the presence of currents, one can use the complexness parameter $q_{n}^{2}=\left\langle\left(\operatorname{Im} \psi_{n}\right)^{2}\right\rangle /\left\langle\left(\operatorname{Re} \psi_{n}\right)^{2}\right\rangle$ introduced by Lobkis and Weaver [51] as the ratio of the variance of the imaginary and real parts of the $n$th resonance state (after a phase rotation leading to independent real and imaginary components [52]). These authors experimentally obtained the complexness parameter from the distribution of the phase $\varphi$ of the spatial component of the field : $\psi=|\psi| e^{i \varphi}$. By considering that the real and imaginary parts of the field are independant Gaussian random variables, they obtained:

$$
P(\varphi)=\frac{q}{2 \pi} \frac{1}{q^{2} \cos ^{2} \varphi+\sin ^{2} \varphi} .
$$

This distribution is closely related to the Poisson kernel (eq. 15) for a given frequency. This expression is peaked around 0 and $\pi$ for purely standing waves in a closed cavity. As losses increase, the phase probability distribution broadens corresponding to a growing traveling-wave component of the mode. The 

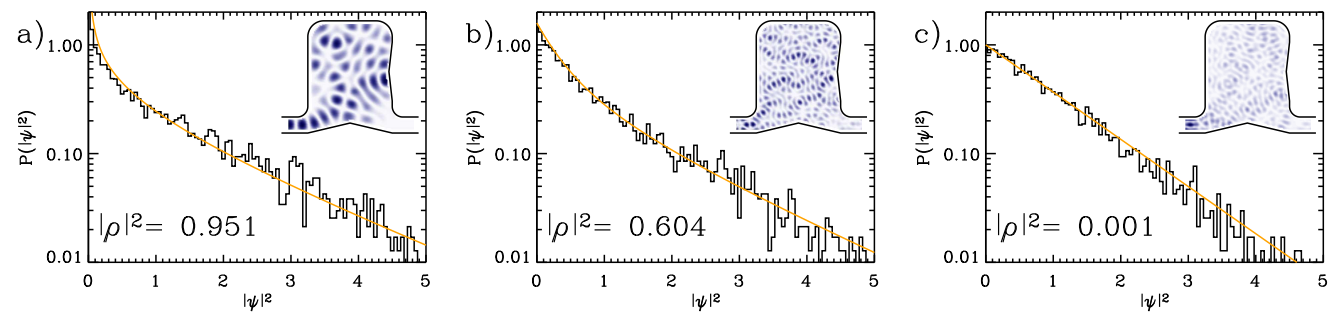

Fig. 6 Distribution of intensities for three different wave functions at frequencies $\nu=8.0$ (a), 16.9 (b), and $15.4 \mathrm{GHz}$ (c) are shown with different phase rigidities $|\rho|^{2}$. The solid line represents the distribution from eq. (28). In the inserts the corresponding modulus of the wave function is shown (see [56]).

latter feature has been recently experimentally verified by means of elastic waves in a chaotic plate [53]. In the context of microwave cavities, a derived distribution had been obtained by J. Barthélemy et al. [54].

A related quantity, namely the phase rigidity $|\rho|^{2}$ can be expressed as a function of the complexness parameter:

$$
|\rho|=\frac{\left\langle\psi_{R}^{2}\right\rangle-\left\langle\psi_{I}^{2}\right\rangle}{\left\langle\psi_{R}^{2}\right\rangle+\left\langle\psi_{I}^{2}\right\rangle}=\frac{1-q^{2}}{1+q^{2}} .
$$

By considering the phase rigidity as a continuous function of frequency, P. Brouwer studied the effect of openness [55] and derived the theoretical distribution of $|\rho|^{2}$, which has been experimentally verified by Kim et al. in an open microwave cavity $[50,56]$.

For fixed phase rigidity $|\rho|^{2}$ the distribution of wave function intensities is given by $[55,57]$

$$
P_{\rho}(I)=\frac{1}{\sqrt{1-|\rho|^{2}}} \exp \left[-\frac{I}{1-|\rho|^{2}}\right] I_{0}\left[\frac{|\rho| I}{1-|\rho|^{2}}\right] \text {. }
$$

For $|\rho|^{2} \rightarrow 1, P_{\rho}$ is approaching a Porter-Thomas, whereas it is an exponential distribution for $|\rho|^{2}=$ 0 . Porter-Thomas and single exponential distributions are $\chi^{2}$ distributions for one and two degrees of freedom, reflecting the fact that in closed systems the wave function is real, whereas in fully open systems it contains a real and an imaginary part of comparable magnitude. In Fig. 6 the distribution of intensities for three different wave functions of an open microwave cavity is shown with different phase rigidity values ranging from nearly closed $\left(|\rho|^{2} \approx 1\right)$ to nearly completely open $\left(|\rho|^{2} \approx 0\right)$. A good agreement is seen, especially as the only parameter, the phase rigidity $|\rho|^{2}$, has been fixed beforehand (for details see [50,56]).

The complexness parameter $q_{n}$ has been investigated both experimentally $[53,54]$ and theoretically $[5,26]$ through the scope of its relationship to the resonance width. In particular, in Ref. [5] the authors have established that the complexness parameter is also a measure of the non-orthogonality of eigenfunctions in the case of inhomogeneous losses.

A linear relationship between $q_{n}$ and $\Gamma_{n}$ was first noticed by Barthélemy et al. [54] analyzing hundreds of resonance states of a 2D chaotic microwave cavity at room temperature. This result was then confirmed using the effective Hamiltonian formalism in the limit $M \gg 1$, relevant in the experiment [5]. A linear relationship between $q_{n}$ and $\Gamma_{n}$ was also verified in an elastodynamics experiment for a given resonance when a spatially extended coupling is varied [53]. The complexness parameter was also investigated at arbitrary $M$ by means of its probability distribution in the regime of weak coupling [26]. There, it was shown that the average value of $q_{n}^{2}$ is directly proportional to the variance of $\Gamma_{n}$, which constitutes the natural measure of the fluctuations of the widths.

In reference [6], the authors confronted theoretical predictions presented in [26] to numerical solutions of the Maxwell equations in a 2D chaotic microwave cavity with lossy boundaries subject to Ohmic dissipation. In the regime of weak coupling, which corresponds to the condition $\sqrt{\operatorname{var}(\Gamma)} \ll \Delta$, where $\Delta$ is the mean level spacing, the anti-Hermitian part of $H_{\text {eff }}$ is small compared to the Hermitian part and perturbation theory can be applied. 

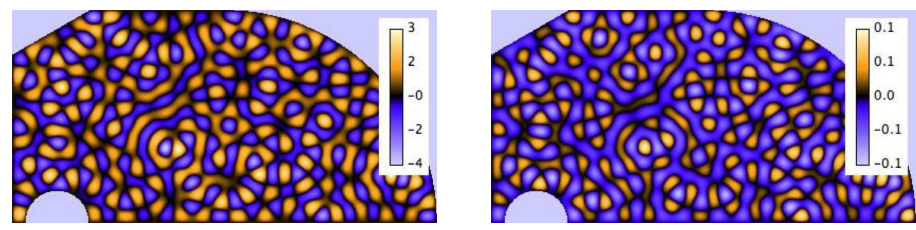

Fig. 7 Real (on the left) and imaginary (on the right) component of the 500th resonance state obtained by means of the Finite Element Method (see [6]).

We define the rescaled widths $\gamma=\pi \Gamma /(2 \kappa \Delta)$ with $\langle\gamma\rangle=M$ where we assume a single and real coupling constant $\kappa$ for the $M$ fictitious channels accounting for absorption. Considering that the widths are given by a sum of $M$ squared independent Gaussian random variables, they follow a $\chi^{2}$ distribution with $M$ degrees of freedom:

$$
\chi_{M}^{2}(\gamma)=\frac{1}{2^{M / 2} \Gamma(M / 2)} \gamma^{M / 2-1} e^{-\gamma / 2}
$$

where $\Gamma(\cdot)$ is the Euler gamma function.

The distribution of the rescaled complexness parameter $X_{n} \equiv q_{n}^{2} / \kappa^{2}$, was obtained by making use of group integral methods [58] and reads:

$$
\mathcal{P}_{M}^{G O E}(X)=\frac{M}{6 X^{2}} \frac{1+(3+M) X^{-1}}{\left(1+X^{-1}\right)^{M / 2+2}},
$$

where strong mode-to-mode fluctuations clearly appear, embodied in the power law tail $1 / X^{2}$ of the distribution.

The chaotic cavity considered in [6] has a quarter of a stadium shape with a radius of $R=1 \mathrm{~m}$ and a length of $l=2 \mathrm{~m}$ (see Fig. 7). In order to reduce the bouncing ball modes between the two parallel sides, an oblique cut is performed on one side and a movable perfectly reflecting half disk of diameter $d=0.3 \mathrm{~m}$ is placed on the opposite side. The absorbing boundary condition is imposed on an adjustable part of the boundary of the cavity. The number of channels $M$ is related to the effective absorbing length $l_{a b s}$ along the boundary and the wavelength $\lambda$ through:

$$
M=\frac{l_{a b s}}{\lambda / 2} .
$$

Note that $M$ is also related to the first two moments of the width distribution (29):

$$
M=\frac{2}{\left\langle\gamma^{2}\right\rangle /\langle\gamma\rangle^{2}-1} .
$$

Fig. 8 shows the distributions of the complexness parameter $X$ compared to the theoretical distribution (30). Three different sets of values of the absorbing lengths, conductivities and frequency ranges are used (see caption). For each frequency interval, the number $M$ of channels used for the comparison corresponds to the nearest integer value of relation (31) computed with the median value of the wavelength in each interval (the estimates (31) and (32) agree within a few percents). The excellent agreement, even in the tail of the distribution (as shown in inset for $M=38$ ), confirms that the prediction (30), obtained within the perturbation theory, contains the essential features to account for the non-orthogonality of the resonance states due to spatially continuously distributed losses.

Note that in the width distributions presented in Subsec. 3.2 in the lower row of Fig. 2, the above presented distributions of the complexness parameter correspond to the additional fluctuating absorption, which must be taken into account to obtain a good agreement between theory and experiment [41]. 

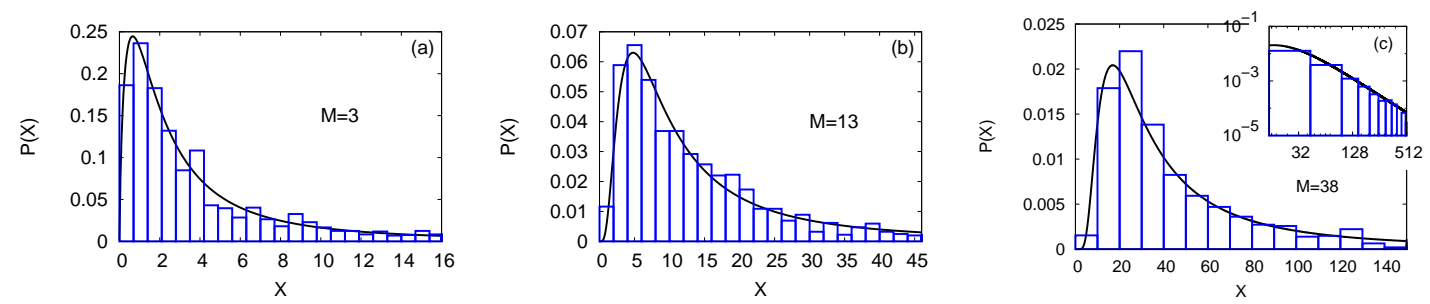

Fig. 8 Distributions of the complexness parameter for different absorbing lengths, conductivities and frequency ranges: (a) $l_{a b s}=\pi / 18, \sigma_{c}=80 \mathrm{Sm}^{-1}$ and from the 300 th to the 400 th resonances; (b) $l_{a b s}=\pi / 6, \sigma_{c}=400 \mathrm{Sm}^{-1}$ and from the 700 th to the 800th resonances; (c) $l_{a b s}=\pi / 2, \sigma_{c}=1000 \mathrm{Sm}^{-1}$ and from the 700 th to the 800th resonances. The histograms show the numerical distributions. The solid lines correspond to the theoretical prediction (30) where the number $M$ of channels corresponds to the nearest integer value of relation (31) computed with the median value of the wavelength in each frequency interval (see [6]).

\section{Summary and Outlook}

We have shown that classical wave experiments are perfectly suited to investigate systems which can be described by the effective Hamiltonian approach. They permit easy access to the full complex scattering matrix, thus giving the possibility to fix relevant parameters beforehand. Additionally the system can be controlled in various ways, like boundary variations on subwavelength scale or coupling variations. This allows to verify experimentally various predictions given by the effective Hamiltonian approach. Since absorption often plays a crucial role for classical waves, it has to be taken into account properly in the theory. Thus, classical experiments in the realm of effective Hamiltonians are a perfect tool to test theoretical predictions and, additionally, are also a driving part in the further development and understanding of effects, induced by the coupling to the environment, i.e. by an effective Hamiltonian.

On the theoretical side, a continuous progress of statistical description of new quantities is going on, where microwave experiments will be one of the major tools to verify these predictions. Recently for example the distribution of 'width velocity' has been predicted by Fyodorov and Savin [59] which should be accessible by microwave experiments. On the other hand experimental findings will trigger further theoretical investigations as it has been the case for including effects of absorption, for local level dynamics [60] and for the coupling fidelity [11]. Experimentally challenging is still the reliable extraction of resonances in the strongly overlapping regime. This would give access to follow the resonances far in the complex plane and investigate nearest neighbor distribution in the complex plane, resonance correlations etc. One could also verify predictions for the fractal Weyl law [61-63] which relates the average number of resonances to the fractal dimension of the repeller of the classical dynamics.

We would like to acknowledge J. Barthélemy, P. Brouwer, B. Dietz, R. Höhmann, B. Köber, G. LunaAcosta, R. A. Méndez-Sánchez, E. Persson, C. Poli, I. Rotter, D. Savin, P. Sebbah, H.-J. Stöckmann, and O. Xeridat for discussion, data acquisition, data evaluation, etc.

\section{References}

[1] I. Rotter, J. Phys. A 42, 153001 (2009).

[2] H. J. Stöckmann, Quantum Chaos - An Introduction (University Press, Cambridge, 1999).

[3] M. Büttiker, Phys. Rev. B 33, 3020 (1986).

[4] A. Bärnthaler, S. Rotter, F. Libisch, J. Burgdörfer, S. Gehler, U. Kuhl, and H. J. Stöckmann, Phys. Rev. Lett. 105, 056801 (2010).

[5] D. V. Savin, O. Legrand, and F. Mortessagne, Europhys. Lett. 76, 774 (2006).

[6] C. Poli, O. Legrand, and F. Mortessagne, Phys. Rev. E 82, 055201(R) (2010).

[7] E. Persson, I. Rotter, H. J. Stöckmann, and M. Barth, Phys. Rev. Lett. 85, 2478 (2000).

[8] H. J. Stöckmann, E. Persson, Y. H. Kim, M. Barth, U. Kuhl, and I. Rotter, Phys. Rev. E 65, 066211 (2002).

[9] R. Höhmann, U. Kuhl, and H. J. Stöckmann, Phys. Rev. Lett. 100, 124101 (2008). 
[10] R. Schäfer, H. J. Stöckmann, T. Gorin, and T. H. Seligman, Phys. Rev. Lett. 95, 184102 (2005).

[11] B. Köber, U. Kuhl, H. J. Stöckmann, T. Gorin, D. V. Savin, and T. H. Seligman, Phys. Rev. E 82, 036207 (2010).

[12] C. Mahaux and H. A. Weidenmüller, Shell-Model Approach to Nuclear Reactions (North-Holland, Amsterdam, 1969).

[13] J. J. M. Verbaarschot, H. A. Weidenmüller, and M. R. Zirnbauer, Phys. Rep. 129, 367 (1985).

[14] V. V. Sokolov and V. G. Zelevinsky, Nucl. Phys. A 504, 562 (1989).

[15] Y. V. Fyodorov and H. J. Sommers, J. Math. Phys. 38, 1918 (1997).

[16] F. M. Dittes, Phys. Rep. 339, 215 (2000).

[17] Y. V. Fyodorov, D. V. Savin, and H. J. Sommers, J. Phys. A 38, 10731 (2005).

[18] T. Guhr, A. Müller-Groeling, and H. A. Weidenmüller, Phys. Rep. 299, 189 (1998).

[19] J. Barthélemy, O. Legrand, and F. Mortessagne, Phys. Rev. E 71, 016205 (2005).

[20] R. A. Méndez-Sánchez, U. Kuhl, M. Barth, C. H. Lewenkopf, and H. J. Stöckmann, Phys. Rev. Lett. 91, 174102 (2003).

[21] U. Kuhl, M. Martínez-Mares, R. A. Méndez-Sánchez, and H. J. Stöckmann, Phys. Rev. Lett. 94, 144101 (2005).

[22] V. V. Sokolov and V. G. Zelevinsky, Ann. Phys. (N.Y.) 216, 323 (1992).

[23] C. Poli, B. Dietz, O. Legrand, F. Mortessagne, and A. Richter, Phys. Rev. E 80, 035204(R) (2009).

[24] J. Zakrzewski and M. Kuś, Phys. Rev. Lett. 67, 2749 (1991).

[25] B. Dietz, A. Heine, A. Richter, O. Bohigas, and P. Leboeuf, Phys. Rev. E 73, 035201(R) (2006).

[26] C. Poli, D. Savin, O. Legrand, and F. Mortessagne, Phys. Rev. E 80, 046203 (2009).

[27] H. Alt, H. D. Gräf, H. L. Harney, R. Hofferbert, H. Lengeler, A. Richter, P. Schardt, and H. A. Weidenmüller, Phys. Rev. Lett. 74, 62 (1995).

[28] C. Dembowski, H. D. Gräf, H. L. Harney, A. Heine, W. D. Heiss, H. Rehfeld, and A. Richter, Phys. Rev. Lett. 86, 787 (2001).

[29] B. Dietz, T. Friedrich, J. Metz, M. Miski-Oglu, A. Richter, F. Schäfer, and C. A. Stafford, Phys. Rev. E 75, 027201 (2007).

[30] U. Günther, I. Rotter, and B. F. Samsonov, J. Phys. A 40, 8815 (2007).

[31] W. D. Heiss, M. Müller, and I. Rotter, Phys. Rev. E 58, 2894 (1998).

[32] G. López, P. A. Mello, and T. H. Seligman, Z. Phys. A 302, 351 (1981).

[33] P. A. Mello, P. Pereyra, and T. H. Seligman, Ann. Phys. (N.Y.) 161, 254 (1985).

[34] W. A. Friedman and P. A. Mello, Ann. Phys. (N.Y.) 161, 276 (1985).

[35] E. Doron and U. Smilansky, Phys. Rev. Lett. 68, 1255 (1992).

[36] P. W. Brouwer, Phys. Rev. B 51, 16878 (1995).

[37] N. Savytskyy, A. Kohler, S. Bauch, R. Blümel, and L. Sirko, Phys. Rev. E 64, 036211 (2001).

[38] G. B. Akguc and L. E. Reichl, Phys. Rev. E 67, 46202 (2003).

[39] D. V. Savin and H. J. Sommers, Phys. Rev. E 69, 035201 (2004).

[40] D. V. Savin, Y. V. Fyodorov, and H. J. Sommers, Phys. Rev. E 63, 035202(R) (2001).

[41] U. Kuhl, R. Höhmann, J. Main, and H. J. Stöckmann, Phys. Rev. Lett. 100, 254101 (2008).

[42] J. Main, Phys. Rep. 316, 233 (1999).

[43] C. Poli, G. A. Luna-Acosta, and H. J. Stöckmann, Phys. Rev. Lett. 108, 174101 (2012).

[44] I. Rotter, Rep. Prog. Phys. 54, 635 (1991).

[45] I. Rotter, Phys. Rev. E 64, 036213 (2001).

[46] R. A. Jalabert and H. M. Pastawski, Phys. Rev. Lett. 86, 2490 (2001).

[47] T. Gorin, T. Prosen, T. H. Seligman, and M. Žnidarič, Phys. Rep. 435, 33 (2006).

[48] H. Schanze, H. J. Stöckmann, M. Martínez-Mares, and C. H. Lewenkopf, Phys. Rev. E 71, 016223 (2005).

[49] R. Pnini and B. Shapiro, Phys. Rev. E 54, 1032(R) (1996).

[50] Y. H. Kim, U. Kuhl, H. J. Stöckmann, and P. W. Brouwer, Phys. Rev. Lett. 94, 036804 (2005).

[51] O. I. Lobkis and R. L. Weaver, J. Acoust. Soc. Am. 108, 1480 (2000).

[52] A. I. Saichev, H. Ishio, A. F. Sadreev, and K. F. Berggren, J. Phys. A 35, L87 (2002).

[53] O. Xeridat, C. Poli, O. Legrand, F. Mortessagne, and P. Sebbah, Phys. Rev. E 80, 035201 (R) (2009).

[54] J. Barthélemy, O. Legrand, and F. Mortessagne, Europhys. Lett. 70, 162 (2005).

[55] P. W. Brouwer, Phys. Rev. E 68, 046205 (2003).

[56] U. Kuhl, Eur. Phys. J. Special Topics 145, 103 (2007).

[57] H. Ishio, A. I. Saichev, A. F. Sadreev, and K. F. Berggren, Phys. Rev. E 64, 056208 (2001).

[58] H. Schomerus, K. M. Frahm, M. Patra, and C. W. J. Beenakker, Physica A 278, 469 (2000).

[59] Y. V. Fyodorov and D. V. Savin, Phys. Rev. Lett. 108, 184101 (2012).

[60] M. Barth, U. Kuhl, and H. J. Stöckmann, Phys. Rev. Lett. 82, 2026 (1999).

[61] J. Sjöstrand and M. Zworski, Comm. in part. diff. eqns 18, 847 (1993).

[62] W. T. Lu, S. Sridhar, and M. Zworski, Phys. Rev. Lett. 91, 154101 (2003).

[63] J. Wiersig and J. Main, Phys. Rev. E 77, 036205 (2008). 